\title{
New copulas obtained by maximizing Tsallis or Rényi Entropies
}

\author{
Doriano-Boris Pougaza and Ali Mohammad-Djafari \\ Laboratoire des Signaux et Systèmes \\ UMR 8506 (CNRS-SUPELEC-UNIV PARIS SUD 11) \\ Plateau de Moulon, 3 rue Joliot Curie, \\ 91192 Gif-sur-Yvette Cedex, France
}

\begin{abstract}
Sklar [1] introduced the notion of copula, solving the problem studied by Fréchet [2] and others on the determination of a joint distribution function when the one dimensional marginal cumulative distributions are prescribed. The same problem also arises in the context of image (the internal density distribution of some physical or biological quantity inside a section of the body) reconstruction in X-ray computated tomography when only two orthogonal projections are given. The two problems are mathematically equivalent when restricted to distributions with bounded support, we propose to study the solutions which maximize Shannon [3], Tsallis-Havrda-Charvát [4, 5] or the Rényi [6] entropies by rescaling. The case of Shannon and Tsallis or Rényi with index $q=2$ admits analytic solutions which curiously give new copula families. In this paper, we give a theorem and its corollary using the wellknown uniform transformation yielding a method for constructing new family of copulas. We also give the expression of some dependence concepts and then provide many examples of this method in practice.
\end{abstract}

Keywords: Copula, Entropy, Joint density estimation, Shannon, Rényi and Tsallis.

PACS: $02.30 . \mathrm{Gp}, 02.50 . \mathrm{Cw}, 02.50 . \mathrm{Sk}$

\section{Introduction}

The problem of computed tomography (CT) is to reconstruct an image $f\left(x_{1}, x_{2}\right)$ which represents the distribution of material density in a section of a body from radiographies, called mathematically projections or line integrals, at different angles around it. The mathematical model relating the projections to the images is the Radon transform [7].

When the number of projections are limited the problem is ill-posed. Here, we consider the particular case where we have only two projections (horizontally $f_{1}\left(x_{1}\right)$ and vertically $f_{2}\left(x_{2}\right)$ ): the mathematical inverse problem is then finding $f\left(x_{1}, x_{2}\right)$ from the knowledge of $f_{1}\left(x_{1}\right)$ and $f_{2}\left(x_{2}\right)$, where $f_{1}\left(x_{1}\right)$ and $f_{2}\left(x_{2}\right)$ are obtained from $f\left(x_{1}, x_{2}\right)$ by integrating over $x_{1}$ and $x_{2}$ respectively.

The inverse problem of determining $f\left(x_{1}, x_{2}\right)$ from its marginals has infinite number of solutions in the form $f\left(x_{1}, x_{2}\right)=f_{1}\left(x_{1}\right) f_{2}\left(x_{2}\right) c\left(x_{1}, x_{2}\right)$ where $c\left(x_{1}, x_{2}\right)$ is called copula density $([8,9,10,11])$. 
There are many families of copulas which differ in the detail of the dependence they represent. A family will typically have several parameters which relate to the strength and form of these dependencies. Looking for a unique solution $f\left(x_{1}, x_{2}\right)$, then is equivalent to looking for a unique copula $c\left(x_{1}, x_{2}\right)$. To well pose the problem, we proposed the use of entropies as regularizers and consequently denote these as maximum entropy copulas.

To position the novelty of our approach and the main results of this paper we report here the main results of those papers that are related closely to our paper. The kind of information that one has in hands can be incorporated in the model of reconstruction in tomography as a constraint optimization problem in the form of different types of entropies which are seen as a measure of disorder, or more precisely unpredictability. In [12, 13], relative entropy is minimized with uniform marginals and a given rank correlation, giving a numerical algorithm for the computation of the resulting distribution. Recently in [14], the authors found a multidimensional checkerboard copula of maximum Shannon's entropy to match an observed set of grade correlation coefficients. The problem was formulated as the maximization of a concave function, then reformulated as an unconstrained minimization, and then solved numerically using a Newton iteration. This does not, however, give closed form multivariate copulas.

In this paper, we consider the cases where we can compute a closed form solution. This is really the novelty of our approach. In particular by considering the Rényi or Tsallis entropy with index $q=2$ and particular expressions for the marginals we obtained new families of copula. There have been many approaches to construct new families of copulas (see for example [11] and references therein): the closest expression we found (to our knowledge) is the copula family obtained in [15], where the author describes a method of constructing a multivariate distribution with given marginals. However, the construction and final result are different.

To be more explicit we summarize the method in [15] as follows :

Find a function $f^{1}(u, v)$ such that

$$
\int_{0}^{1} \int_{0}^{1} f^{1}(u, v) d u d v=0 \text { and } \int_{0}^{1} f^{1}(u, v) d u=0, \int_{0}^{1} f^{1}(u, v) d v=0,
$$

then compute any function $f$ whose integrals, defined by

$$
\begin{gathered}
\int_{0}^{1} \int_{0}^{1} f(u, v) d u d v=\Delta \\
\int_{0}^{1} f(u, v) d v=f_{1}(u), \text { and } \int_{0}^{1} f(u, v) d u=f_{2}(v),
\end{gathered}
$$

exist and are finite, giving

$$
f^{1}(u, v)=f(u, v)-f_{1}(u)-f_{2}(v)+\Delta .
$$

Then, using the joint distribution $f(u, v)$ and its marginals $f_{1}(u)$ and $f_{2}(v)$, the author obtains the following copula density family :

$$
c(u, v)=1+\theta f^{1}(u, v)
$$

for a suitable parameter $\theta$ which is introduced if the constraint of positivity in equation (4) is not satisfied but $f^{1}$ is bounded. In general $1+\sum_{i=1}^{n} f_{i}^{1}$ is a density when the $f_{i}^{1}$ fulfill the conditions (1). This method allows to generate all polynomial copula [16]. 
The approach we propose is different. We instead consider the probability density function $f\left(x_{1}, x_{2}\right)$ and its marginals $f_{1}\left(x_{1}\right)$ and $f_{2}\left(x_{2}\right)$ :

$$
\left\{\begin{array}{l}
\int_{0}^{1} f\left(x_{1}, x_{2}\right) d y=f_{1}\left(x_{1}\right), \quad \forall x_{1} \\
\int_{0}^{1} f\left(x_{1}, x_{2}\right) d x=f_{2}\left(x_{2}\right), \quad \forall x_{2} \\
\int_{0}^{1} \int_{0}^{1} f\left(x_{1}, x_{2}\right) d x_{1} d x_{2}=1, \\
f\left(x_{1}, x_{2}\right) \geqslant 0,
\end{array}\right.
$$

and determine a unique solution for $f\left(x_{1}, x_{2}\right)$. But as this problem is ill posed we regularize it by looking for the unique solution (if it exists) that maximizes an entropy. As we have showed before [17] when the Shannon entropy is used we obtained $f_{1}\left(x_{1}, x_{2}\right)=f_{1}\left(x_{1}\right) f_{2}\left(x_{2}\right)$ which corresponds to uniform copula. When using the Rényi's or Tsallis entropy when $q=2$ we obtained new families.

This paper finalizes our main results and is structured as follows:

First, we give the definition of distributions with bounded support. Then we state our theorem for constructing new $n$-dimensional maximum entropy distributions given only its univariate marginals, and a new constructive way to generate new copula by using Sklar's theorem. In the bivariate case, we give expression of some dependence concepts such as the Kendall's tau $(\tau)$ coefficient, the Spearman's rho $(\rho)$ and the Gini's gamma $\left(\gamma_{C}\right)[11,18,19]$. Finally some examples of family of copulas are given using tractable closed form expressions of the inverse cumulative distributions such as the standard Kumaraswamy distribution and the two-sided power distributions. To our knowledge, the examples of family of copula we derived are different in their statistical properties from all known families of copula and they are new.

\section{Preliminaries}

We refer to the book by A.W. Marshall [20] for the definition of distribution with bounded support:

Definition 2.1 Distributions that have support contained in a known finite interval can be translated using scale and location parameters so that the support of the distribution is contained in the interval $\mathbf{I}=[0,1]$, but in no closed subinterval of $\mathbf{I}$. More precisely, these are distributions $F$ of nonnegative random variables $T$ that can take values arbitrarily close to 0 and 1 , but have the property that $P\{0 \leq T \leq 1\}=1$. Such distributions are identified by the conditions

$$
F(0-)=0, \quad 0<F(t)<1 \text { for } 0<t<1, \quad \text { and } F(1)=1 .
$$

$F(0-)$ holds for the left one-sided limit at 0.

Letting $f\left(x_{1}, x_{2}\right)$ be the joint probability density function (pdf) on $[0,1]^{2}$. We consider two measures of entropy, namely: 
(i) Tsallis-Havrda-Charvat's entropy $[4,5]$

$$
H=\frac{1}{1-q}\left(1-\int_{0}^{1} \int_{0}^{1} f^{q}\left(x_{1}, x_{2}\right) d x_{1} d x_{2}\right), q \geqslant 0 \text { and } q \neq 1
$$

(ii) Rényi entropy $[6]$

$$
R=\frac{1}{1-q} \ln \left(\int_{0}^{1} \int_{0}^{1} f^{q}\left(x_{1}, x_{2}\right) d x_{1} d x_{2}\right), q \geqslant 0 \text { and } q \neq 1 .
$$

Note that they are related,

$$
R=\frac{1}{1-q} \ln [(q-1) H+1], H=\frac{1}{1-q}\left[1-e^{(1-q) R}\right],
$$

so that $R$ and $H$ achieve the same maxima $\forall q$.

\section{Main Results}

The following are the main results of this paper.

Theorem 3.1 Let $F_{i}, i=1, \ldots, n$ be absolutely continuous one dimensional marginal cumulative distribution functions (cdf's) with domain $\mathbf{I}$ of an absolutely continuous n-dimensional cumulative distribution $F$ with domain $\mathbf{I}^{n}$. If $F$ maximizes Tsallis' entropy (or the Rényi's entropy by rescaling) when the entropy index equals 2 , then $F$ is given by

$$
F\left(x_{1}, \ldots, x_{n}\right)=\sum_{i=1}^{n} F_{i}\left(x_{i}\right) \prod_{\substack{j=1 \\ j \neq i}}^{n} x_{j}+(1-n) \prod_{i=1}^{n} x_{i} .
$$

The proof in the bivariate case and the extension to the multivariate case is straightforward and can be found in [17].

Combining Theorem 3.1 with the inversion method (See corollary 2.3.7 in [11], p. 22), we deduce the following corollary which gives a new method of constructing a family of copulas.

Corollary 3.2 Let $F_{i}, i=1, \ldots, n$ be as in Theorem 3.1, and let $F_{i}^{(-1)}$ be quasi-inverses (See definition 2.3.6 in [11], p. 21) of $F_{i}$. Then for any $\boldsymbol{u}$ in $\mathbf{I}^{n}$,

$$
C\left(u_{1}, \ldots, u_{n}\right)=\max \left\{\sum_{i=1}^{n} u_{i} \prod_{\substack{j=1 \\ j \neq i}}^{n} F_{j}^{(-1)}\left(u_{j}\right)+(1-n) \prod_{i=1}^{n} F_{i}^{(-1)}\left(u_{i}\right), 0\right\}
$$

is a copula.

Theorem 3.1 can thus be used as a constructive method for determining a joint multivariate distribution from the only knowledge of its marginals and the Corollary 3.2 as a tool for creating families of copula just by specifying marginals. This is what we are going to present for the bivariate case in the next section. 
In the bivariate case,

$$
C(u, v)=v \Phi(u)+u \psi(v)-\Phi(u) \psi(v)
$$

where $F_{1}^{-1}\left(u_{1}\right) \equiv \Phi$ and $F_{2}^{-1}\left(u_{2}\right) \equiv \psi$, for any univariate functions $\Phi$ and $\psi$, the following properties:

(1) $\Phi(0)=\psi(0)=0$,

(2) $\Phi(1)=\psi(1)=1$.

Therefore to determine the case in which $C$ is a copula the result from [21] can be adapted. We may also remark that in [21], the authors have proposed the following family:

$$
C(u, v)=u v+\theta \Phi(u) \psi(v), \quad \theta \in[-1,1]
$$

where $\Phi, \psi$ are absolutely continuous distributions on $[0,1]$ and their derivatives are bounded for almost every values taken in $[0,1]$, with the conditions

(1) $\Phi(0)=\psi(0)=0$,

(2) $\Phi(1)=\psi(1)=0$.

Family (12) generalizes many well-known copulas but are different from families of copulas (11) we derived using our method. In particular, if $\theta=-1$ and $u v=v \Phi(u)+u \psi(v)$ the two families coincide. But to see the differences one may compute and then compare some of their statistical properties, for example their dependencies measures. One first remark that in our case $\Phi \equiv F_{1}^{-1}\left(u_{1}\right)$ and $\psi \equiv F_{2}^{-1}\left(u_{2}\right)$ but in (12), the functions $\Phi$ and $\psi$ do not have these remarkable interpretations.

Solving the following system of inequalities (See Theorem 2.2.7 in [11], p. 13) yields the conditions on $\Phi$ and $\psi$, so that (11) satisfies all the properties of a copula for any $v$ (resp. $u$ ) in $[0,1]$ such that the partial derivative $C_{u}$ (resp. $C_{v}$ ) exists, for almost all $u$ (resp. $v$ ), and for such $u$ and $v$ we have respectively :

$$
\left\{\begin{array}{l}
0 \leqslant \frac{\partial C(u, v)}{\partial u} \leqslant 1 \\
0 \leqslant \frac{\partial C(u, v)}{\partial v} \leqslant 1
\end{array}\right.
$$

From Eq.(11):

$$
\frac{\partial C(u, v)}{\partial u}=\Phi^{\prime}(u)(v-\psi(v))+\psi(v)
$$

Similarly

$$
\frac{\partial C(u, v)}{\partial v}=\psi^{\prime}(v)(u-\Phi(u))+\Phi(u)
$$

In particular when $\psi(v)=v$ or $\Phi(u)=u$, we have the independent copula $C(u, v)=u v$ which is a member of the family of copula (11), and also the function $\psi$ or $\Phi$ is always in $[0,1]$.

In general, there are four different cases to consider in order that (11) be a copula depending on the choice of the functions $\psi$ and $\Phi$ :

Case 1: If $\psi(v)<v($ resp. if $\Phi(u)<u)$ we have respectively :

$$
\left\{\begin{array}{l}
0 \leqslant \Phi^{\prime}(u)(v-\psi(v))+\psi(v) \leqslant 1 \\
0 \leqslant \psi^{\prime}(v)(u-\Phi(u))+\Phi(u) \leqslant 1 .
\end{array}\right.
$$


Then

$$
\left\{\begin{array}{l}
0 \leqslant \Phi^{\prime}(u)+\frac{\psi(v)}{v-\psi(v)} \leqslant \frac{1}{v-\psi(v)} \\
0 \leqslant \psi^{\prime}(v)+\frac{\Phi(u)}{u-\Phi(u)} \leqslant \frac{1}{u-\Phi(u)},
\end{array}\right.
$$

therefore

$$
\left\{\begin{array}{l}
0 \leqslant \Phi^{\prime}(u)+\frac{\psi(v)}{v-\psi(v)} \leqslant \frac{1}{v-\psi(v)} \\
0 \leqslant \psi^{\prime}(v)+\frac{\Phi(u)}{u-\Phi(u)} \leqslant \frac{1}{u-\Phi(u)} .
\end{array}\right.
$$

Finally, we have

$$
\begin{aligned}
& \left\{\begin{array}{l}
0 \leqslant \Phi^{\prime}(u)+\frac{\psi(v)}{v-\psi(v)} \\
0 \leqslant \frac{1-\psi(v)}{v-\psi(v)}-\Phi^{\prime}(u)
\end{array}\right. \\
& \left\{\begin{array}{l}
0 \leqslant \psi^{\prime}(v)+\frac{\Phi(u)}{u-\Phi(u)} \\
0 \leqslant \frac{1-\Phi(u)}{u-\Phi(u)}-\psi^{\prime}(v) .
\end{array}\right.
\end{aligned}
$$

Case 2: If $\psi(v)>v$ (or resp. if $\Phi(u)>u$ ) we have respectively:

$$
\left\{\begin{array}{l}
0 \leqslant \Phi^{\prime}(u)(v-\psi(v))+\psi(v) \leqslant 1 \\
0 \leqslant \psi^{\prime}(v)(u-\Phi(u))+\Phi(u) \leqslant 1 .
\end{array}\right.
$$

Then

$$
\left\{\begin{array}{l}
-\psi(v) \leqslant \Phi^{\prime}(u)(v-\psi(v)) \leqslant 1-\psi(v) \\
-\Phi(u) \leqslant \psi^{\prime}(v)(u-\Phi(u)) \leqslant 1-\Phi(u) .
\end{array}\right.
$$

Therefore

Finally, we have

$$
\left\{\begin{array}{l}
\frac{1-\psi(v)}{v-\psi(v)} \leqslant \Phi^{\prime}(u) \leqslant \frac{-\psi(v)}{v-\psi(v)} \\
\frac{1-\Phi(u)}{u-\Phi(u)} \leqslant \psi^{\prime}(v) \leqslant \frac{-\Phi(u)}{u-\Phi(u)}
\end{array}\right.
$$

$$
\left\{\begin{array}{l}
0 \leqslant \frac{-\psi(v)}{v-\psi(v)}-\Phi^{\prime}(u) \\
0 \leqslant \Phi^{\prime}(u)-\frac{1-\psi(v)}{v-\psi(v)} \\
0 \leqslant \frac{-\Phi(u)}{u-\Phi(u)}-\psi^{\prime}(v) \\
0 \leqslant \psi^{\prime}(v)-\frac{1-\Phi(u)}{u-\Phi(u)} .
\end{array}\right.
$$

To make our computation more compact, we will denote by:

$$
A_{1}(u)=\frac{\Phi(u)}{u-\Phi(u)}, A_{2}(u)=\frac{1-\Phi(u)}{u-\Phi(u)},
$$


and

$$
B_{1}(v)=\frac{\psi(v)}{v-\psi(v)}, B_{2}(v)=\frac{1-\psi(v)}{v-\psi(v)} .
$$

The following table summarizes all the conditions :

\begin{tabular}{|c|c|c|c|}
\hline case 1 & case 2 & case 3 & case 4 \\
\hline$\forall u$ if $\Phi(u)<u$ & $\forall v$ if $\psi(v)<v$ & $\forall u$ if $\Phi(u)>u$ & $\forall v$ if $\psi(v)>v$ \\
\hline $0 \leqslant \psi^{\prime}(v)+A_{1}(u)$ & $0 \leqslant \Phi^{\prime}(u)+B_{1}(v)$ & $0 \leqslant-\psi^{\prime}(v)-A_{1}(u)$ & $0 \leqslant-\Phi^{\prime}(u)-B_{1}(v)$ \\
$0 \leqslant-\psi^{\prime}(v)+A_{2}(u)$ & $0 \leqslant-\Phi^{\prime}(u)+B_{2}(v)$ & $0 \leqslant \psi^{\prime}(v)-A_{2}(u)$ & $0 \leqslant \Phi^{\prime}(u)-B_{2}(v)$ \\
\hline
\end{tabular}

\section{Measures of dependency}

The classical linear dependence between two variables $X$ and $Y$ is the Pearson correlation defined as :

$$
\rho=\frac{\operatorname{cov}(X, Y)}{\sqrt{\operatorname{var}(X)} \sqrt{\operatorname{var}(Y)}} .
$$

The two other most commonly used nonparametric measures of association for two random variables are Spearman's rho $\left(\rho_{S}\right)$ and Kendall's tau $(\tau)([11,22])$. For many joint distributions these measures have different values, as they measure different aspects of the dependence structure. For example, if $X$ and $Y$ are random variables with marginal distribution functions $F_{1}$ and $F_{2}$, respectively, then Spearman's $\rho$ is the ordinary (Pearson) correlation coefficient of the transformed random variables $F_{1}(X)$ and $F_{2}(Y)$, it is defined as

$$
\rho=\frac{\operatorname{cov}\left(F_{1}(X), F_{2}(Y)\right)}{\sqrt{\operatorname{var}\left(F_{1}(X)\right)} \sqrt{\operatorname{var}\left(F_{2}(Y)\right)}}
$$

which is equivalent to :

$$
\rho=12 \int_{0}^{1} \int_{0}^{1} C(u, v) d u d v-3
$$

where $C u, v)$ is their corresponding copula. The Kendall's $\tau$ is the difference between the probability of concordance

$$
P\left[\left(X_{1}-X_{2}\right)\left(Y_{1}-Y_{2}\right)>0\right]
$$

and the probability of discordance

$$
P\left[\left(X_{1}-X_{2}\right)\left(Y_{1}-Y_{2}\right)<0\right]
$$

for two independent pairs $\left(X_{1}, Y_{1}\right)$ and $\left(X_{2}, Y_{2}\right)$ of observations drawn from the distribution, in terms of the copula $C$

$$
\tau=4 \int_{0}^{1} \int_{0}^{1} C(u, v) d C(u, v)-1 .
$$

In terms of dependence properties, Spearman's $\rho$ is a measure of average quadrant dependence, while Kendall's $\tau$ is a measure of average likelihood ratio dependence.

There are also other important concepts of dependence $[18,19]$. One last example we mention here is the Gini's gamma $\left(\gamma_{C}\right)$ :

$$
\gamma_{C}=4 \int_{0}^{1}[C(u, u)+C(u, 1-u)] d u-2 .
$$


As a comparison, one can show that the Spearman's $\rho$ can also be written as

$$
\rho=3 \int_{0}^{1} \int_{0}^{1}\left[(u+v-1)^{2}-(u-v)^{2}\right] d C(u, v)
$$

and the Gini's measure

$$
\gamma_{C}=2 \int_{0}^{1} \int_{0}^{1}[|u+v-1|-|u-v|] d C(u, v) .
$$

\subsection{Expression of depencence measure of our copula family}

After tedious simple algebra, their respective expressions the $n=2$ case of (10) gives :

$$
\tau=4\left(\int_{0}^{1} F_{1}\left(x_{1}\right) d x_{1}-2 \int_{0}^{1} F_{1}\left(x_{1}\right) d x_{1} \int_{0}^{1} F_{2}\left(x_{2}\right) d x_{2}+\int_{0}^{1} F_{2}\left(x_{2}\right) d x_{2}\right)-2,
$$

and

$$
\rho=6\left(\int_{0}^{1} F_{1}\left(x_{1}\right) d x_{1}-2 \int_{0}^{1} F_{1}\left(x_{1}\right) d x_{1} \int_{0}^{1} F_{2}\left(x_{2}\right) d x_{2}+\int_{0}^{1} F_{2}\left(x_{2}\right) d x_{2}\right)-3 .
$$

If we denote by

$$
\xi\left(F_{1}, F_{2}\right)=\int_{0}^{1} F_{1}\left(x_{1}\right) d x_{1}-2 \int_{0}^{1} F_{1}\left(x_{1}\right) d x_{1} \int_{0}^{1} F_{2}\left(x_{2}\right) d x_{2}+\int_{0}^{1} F_{2}\left(x_{2}\right) d x_{2},
$$

we have the following relation between the Kendall's tau and the Spearman's rho coefficients

$$
2 \xi\left(F_{1}, F_{2}\right)-1=\frac{\tau}{2}=\frac{\rho}{3} \text { which implies } \tau=\frac{2}{3} \rho .
$$

And we have also

$$
\gamma_{C}=4\left(\int_{0}^{1} t(\psi(t)+\psi(1-t)) d t+\int_{0}^{1} \phi(t)(1-\psi(t)+\psi(1-t)) d t\right)-2 .
$$

\subsection{Expression of depencences measures for copula in Equation (12)}

We recall the expression obtained in $[21]$ ( $\theta=1$ in Equation (12)):

$$
\begin{gathered}
\tau=8 \int_{0}^{1} \psi(t) d t \int_{0}^{1} \phi(t) d t \\
\rho=12 \int_{0}^{1} \psi(t) d t \int_{0}^{1} \phi(t) d t=\frac{3 \tau}{2} \\
\gamma_{C}=4 \int_{0}^{1} \phi(t)(\psi(t)+\psi(1-t)) d t
\end{gathered}
$$

It is an obvious fact that now our families does not belong to the set of copulas studied by Rodriguez-Lallena and Úbeda-Flores [21] which generalizes many known families of copulas such as the Fairlie-Gumbel-Morgenstern (FGM). Up to our knowledge the families of copulas we have derived in this paper have not discussed in and are new. 


\section{Examples of family of bivariate copula}

In [17] we used the Beta distribution in the case where the parameters allow explicit expressions of the inverse cumulative distributions to obtain examples of families of copula. Here are some other examples of bounded distribution on $[0,1]$ and explicit expression of the inverse cumulative distribution $F_{i}^{-1}\left(x_{i}\right), \quad i=1,2$ which, through the Corollary 3.2 are used to construct other families of copulas.

\subsection{Kumaraswamy distribution}

The probability distribution function (pdf) of the standard Kumaraswamy distribution [23] having nice properties and more tractable than the Beta distribution as it was presented in [24] is given by

$$
f_{i}\left(x_{i}\right)=a b x_{i}^{a-1}\left(1-x_{i}^{a}\right)^{b-1}
$$

with its cdf

$$
F_{i}\left(x_{i}\right)=1-\left(1-x_{i}^{a}\right)^{b}
$$

and inverse cdf

$$
F_{i}^{-1}\left(u_{i}\right)=1-\left(1-u_{i}^{\frac{1}{b}}\right)^{\frac{1}{a}}
$$

where $a>0$ and $b>0$.

Therefore the associated copula for suitable parameters $a$ and $b$ and having two given marginal distributions which are the standard Kumaraswamy distributions, has the following form :

$$
\begin{array}{r}
C\left(u_{1}, u_{2}\right)=u_{1}\left(1-\left(1-u_{2}^{\frac{1}{b}}\right)^{\frac{1}{a}}\right)+u_{2}\left(1-\left(1-u_{1}^{\frac{1}{b}}\right)^{\frac{1}{a}}\right) \\
-\left(1-\left(1-u_{1}^{\frac{1}{b}}\right)^{\frac{1}{a}}\right)\left(1-\left(1-u_{2}^{\frac{1}{b}}\right)^{\frac{1}{a}}\right)
\end{array}
$$

\subsection{Two-sided power distribution}

The pdf of the standard two-sided power distributions introduced in [25] is given by

$$
f_{i}\left(x_{i} ; a, b\right)= \begin{cases}b\left(\frac{x_{i}}{a}\right)^{b-1}, & 0 \leq x_{i} \leq a \\ b\left(\frac{1-x_{i}}{1-a}\right)^{b-1}, & a \leq x_{i} \leq 1\end{cases}
$$

where $0 \leq a \leq 1, b>0$, and its cdf is

$$
F_{i}\left(x_{i} ; a, b\right)= \begin{cases}a\left(\frac{x_{i}}{a}\right)^{b}, & 0 \leq x_{i} \leq a \\ 1-(1-a)\left(\frac{1-x_{i}}{1-a}\right)^{b}, & a \leq x_{i} \leq 1\end{cases}
$$

and evidently

$$
F_{i}^{-1}\left(u_{i} ; a, b\right)= \begin{cases}\sqrt[b]{a u_{i}^{b-1}}, & 0 \leq u_{i} \leq a \\ 1-\sqrt[b]{\left(1-u_{k}\right)(1-a)^{b-1}}, & a \leq u_{i} \leq 1\end{cases}
$$


when $b=2$ it corresponds to the triangular distribution, with the quantile distribution

$$
F_{i}^{-1}\left(u_{i} ; a\right)= \begin{cases}\sqrt{a u_{i}}, & 0 \leq u_{i} \leq a \\ 1-\sqrt{\left(1-u_{i}\right)(1-a)}, & a \leq u_{i} \leq 1\end{cases}
$$

Therefore the associated copula for suitable parameters $a$ and $b$ and having two given marginal distributions which are the two-sided power distributions, has the following form :

Case 1: $0 \leq u_{i} \leq a$ and $b>0$ :

$$
C\left(u_{1}, u_{2}\right)=u_{1}\left(\sqrt[b]{a u_{2}^{b-1}}\right)+u_{2}\left(\sqrt[b]{a u_{1}^{b-1}}\right)-\left(\sqrt[b]{a u_{1}^{b-1}}\right)\left(\sqrt[b]{a u_{2}^{b-1}}\right)
$$

Case 2: $a \leq u_{i} \leq 1$ and $b>0$ :

$$
\begin{aligned}
C\left(u_{1}, u_{2}\right) & =u_{1}\left(1-\sqrt[b]{\left(1-u_{2}\right)(1-a)^{b-1}}\right)+u_{2}\left(1-\sqrt[b]{\left(1-u_{1}\right)(1-a)^{b-1}}\right) \\
& -\left(1-\sqrt[b]{\left(1-u_{1}\right)(1-a)^{b-1}}\right)\left(1-\sqrt[b]{\left(1-u_{2}\right)(1-a)^{b-1}}\right)
\end{aligned}
$$

Up to our knowledge, copulas defined in (38), (43), (44) are new families of copulas. However we have to check for the explicit range of their parameters. We plan to use these copulas for different applications such as criteria for Blind Source Separation and for analyzing multivariate signals.

\section{Conclusion}

We have presented a method which can be used for constructing a multivariate joint distribution from the only knowledge of its univariate marginals, constructing new families of copula. Then we show some families of copula using several distributions with bounded support. Any computer algebra system can be used to figure out the appropriate range of the values of the parameters of these new families of copula. We are still working on this aspect of the problem, but this part is out the aim of this paper, which is to show this method of constructing copula. Indeed, for the bivariate case, the expression and relationship between the Kendall's tau $(\tau)$ and Spearman's rho $(\rho)$ for this new families of copula which are dependencies measure of association between two continuous random variables are shown.

\section{References}

[1] A. Sklar. Fonctions de répartition à n dimensions et leurs marges. Publ. Inst. Statist. Univ. Paris 8, pages 229-231, 1959.

[2] M. Fréchet. Sur les tableaux de corrélation dont les marges sont données. Ann. Univ. Lyon, III. Sér., Sect. A, 14:53-77, 1951.

[3] C.E. Shannon. A mathematical theory of communication. Bell System Technical Journal, $27: 432-379,1948$. 
[4] J. Havrda and F. Charvát. Quantification method of classification processes. Kybernetika, 3(1):30-35, 1967.

[5] C. Tsallis. Possible generalization of Boltzmann-Gibbs statistics. Journal of statistical physics, 52(1):479-487, 1988.

[6] A. Rényi. On measures of dependence. Acta Mathematica Hungarica, 10(3):441-451, 1959.

[7] A. Markoe. Analytic Tomography. Cambridge University Press, 2006.

[8] G. Dall'Aglio, S. Kotz, and G. Salinetti. Advances in probability distributions with given marginals: beyond the copulas. Kluwer Academic Publ., Dordrecht, 1991.

[9] C. Genest and L.P. Rivest. Statistical inference procedures for bivariate archimedean copulas. Journal of the American Statistical Association, 88(423):1034-1043, 1993.

[10] H. Joe. Multivariate Models and Dependence Concepts. London: Chapman \& Hall, 1997.

[11] Roger B. Nelsen. An Introduction to Copulas. Springer Verlag, 2nd edition, 2006.

[12] A.M.H. Meeuwissen and T. Bedford. Minimally informative distributions with given rank correlation for use in uncertainty analysis. Journal of Statistical Computation and Simulation, 57(1):143-174, 1997.

[13] E. Pasha and S. Mansoury. Determination of Maximum Entropy Multivariate Probability Distribution under some Constraints. Applied Mathematical Sciences, 2(57):2843-2849, 2008.

[14] J. Piantadosi, P. Howlett, and J. Borwein. Copulas with Maximum Entropy. Optimization Letters, 5:1-27, 2011.

[15] L. Rüschendorf. Construction of multivariate distributions with given marginals. Annals of the Institute of Statistical Mathematics, 37(1):225-233, 1985.

[16] C.D. Lai and M. Xie. A new family of positive quadrant dependent bivariate distributions. Statistics $\&$ probability letters, 46(4):359-364, 2000.

[17] D.-B. Pougaza and A. Mohammad-Djafari. Maximum entropies copulas. In Proceedings of the 30th International Workshop on Bayesian Inference and Maximum Entropy Methods in Science and Engineering, volume 1305, pages 329-336. American Institute of Physics, 2011.

[18] Roger B. Nelsen. Concordance and copulas: A survey. Distributions with given marginals and statistical modelling, pages 169-178, 2002.

[19] F. Schmid, R. Schmidt, T. Blumentritt, S. Gaißer, and M. Ruppert. Copula-based measures of multivariate association. In Copula Theory and Its Applications: Proceedings of the Workshop Held in Warsaw, 25-26 September 2009, volume 198, page 209. Springer Verlag, 2010 .

[20] A.W. Marshall and I. Olkin. Life distributions: Structure of nonparametric, semiparametric, and parametric families. Springer Verlag, 2007. 
[21] J.A. Rodríguez-Lallena and M. Úbeda-Flores. A new class of bivariate copulas. Statistics 85 probability letters, 66(3):315-325, 2004.

[22] G. A. Fredricks and Roger B. Nelsen. On the relationship between spearman's rho and kendall's tau for pairs of continuous random variables. Journal of Statistical Planning and Inference, 137:2143-2150, 2007.

[23] P. Kumaraswamy. A generalized probability density function for double-bounded random processes. Journal of Hydrology, 46(1-2):79-88, 1980.

[24] M.C. Jones. Kumaraswamy's distribution: A beta-type distribution with some tractability advantages. Statistical Methodology, 6(1):70-81, 2009.

[25] J.R. Van Dorp and S. Kotz. The Standard Two-Sided Power Distribution and its Properties. The American Statistician, 56(2):90-99, 2002. 